Revue d'histoire de l'enfance " irrégulière »

Le Temps de l'histoire

$21 \mid 2019$

L'étrange étrangeté des dossiers de l'enfance irrégulière

\title{
Exercices de style sur " un cas mental et éducatif »
}

Exercices in style on a "mental and educational case"

Mathias Gardet

\section{OpenEdition}

Journals

Édition électronique

URL : https://journals.openedition.org/rhei/4721

DOI : $10.4000 /$ rhei. 4721

ISSN : $1777-540 \mathrm{X}$

Éditeur

Presses universitaires de Rennes

Édition imprimée

Date de publication : 12 novembre 2019

Pagination : $51-66$

ISBN : 978-2-7535-7844-9

ISSN : 1287-2431

Référence électronique

Mathias Gardet, «Exercices de style sur « un cas mental et éducatif » », Revue d'histoire de l'enfance " irrégulière » [En ligne], 21 | 2019, mis en ligne le 12 novembre 2021, consulté le 05 décembre 2022.

URL : http://journals.openedition.org/rhei/4721 ; DOI : https://doi.org/10.4000/rhei.4721

Ce document a été généré automatiquement le 5 décembre 2022.

Tous droits réservés 


\title{
Exercices de style sur « un cas mental et éducatif »
}

\author{
Exercices in style on a "mental and educational case"
}

Mathias Gardet

\section{Rappel des faits}

1 Lucien [le prénom ici a été changé] est né dans le quartier de la Plaine de Plainpalais à Genève le 18 novembre 1923. Il est signalé comme protestant et il est rappelé sans cesse qu'il est bernois d'origine, de la commune de Büren. De façon surprenante, dans presque tous les papiers officiels, figure à la rubrique « Nationalité »: Berne... Au sein de la Suisse, terre fédérale, chaque canton serait-il donc un petit pays? Les barrières linguistiques et culturelles, voire religieuses, semblent en tout cas tenaces: plus tard, on menacera même Lucien, s'il n'est pas sage, de l'envoyer en Suisse allemande ${ }^{1}$ !

2 Son père est menuisier, mais devient par la suite chômeur, sa mère est lavandière et de santé fragile. Il a un frère de trois ans son aîné, Georges, atteint d'un handicap mental. Placé tout d'abord comme externe dans un foyer, ce dernier intègre plus tard comme semi-externe une institution spéciale : l'école-jardin de Varembé. Il sera ensuite mis en famille d'accueil et occupera un emploi de mécanicien. La famille vit dans un troispièces (une cuisine et deux chambres), un rez-de-chaussée sur cour. Lucien partage le même lit que son frère, mais ils ont leur propre chambre. Après être allé à la crèche de Plainpalais jusqu'à ses six ans, Lucien fait pendant deux ans, de 1928 à 1930, sa scolarité primaire dans une école du quartier. Au cours de l'année 1930, du fait de ses mauvais résultats, il est signalé par le directeur de l'école au service médico-pédagogique comme « instable, agité et bagarreur » et est transféré dans une autre école primaire dans une classe de $1^{\text {re }}$ faible. Il y reste presque 4 ans. À cause de son absentéisme, de ses mauvais résultats et de son comportement, il est placé le 5 mars 1934 comme semiexterne à l'école-jardin de Varembé, où se trouve déjà son grand frère. Il y reste jusqu'au 3 février 1936, malgré les menaces d'expulsion pour non-paiement de la pension et fréquentation jugée trop assidue des salles de cinéma. Dès le 17 janvier de la 
même année, Lucien avait en effet été à nouveau l'objet d'un signalement du service médico-pédagogique et, le 29 janvier, il avait été déféré devant la Chambre pénale de l'enfance, accusé de complicité de vol, tout d'abord d'un rouleau de billets d'entrée dans la caisse du Palais des Attractions puis, le lendemain, à l'arraché, d'un sac à main [un « réticule » disait-on à l'époque pour ce type d'accessoire]. Sur décision du tribunal, un internement immédiat pour une durée d'un an est ordonné. Il est alors placé le 4 février 1936 à l'internat « la Maison des Charmilles", dans le quartier des Franchises. $\mathrm{Du}$ fait de la très nette amélioration de son attitude et de son rendement scolaire, il est considéré comme "amendé » et libéré par anticipation le jour de Noël de la même année. Il réintègre, dès la rentrée de janvier 1937, son école primaire, mais cette fois-ci en $5^{\mathrm{e}}$ année normale. Ce retour s'annonce d'emblée difficile, Lucien semble préférer les matchs de boxe et le cinéma. En avril 1937, il est hospitalisé pour une scarlatine. La maladie semble avoir atteint le système moteur de ses jambes, il a du mal à marcher et doit suivre un traitement à l'Institut de physiothérapie. À la rentrée 1937, il est orienté enfin à l'école de préapprentissage du Grütli dirigée par Robert Dottrens, bien qu'il préférerait travailler; en novembre il est d'ailleurs employé comme commissionnaire pour un marchand de graines. En février 1938, il est finalement expulsé pour «mauvaise conduite persistante » et «tentatives d'importuner » les filles de l'école du quartier bien plus chic de Champel qu'il va régulièrement visiter à bicyclette. Appréhendé sur le fait par les gendarmes et toujours suivi par la Chambre pénale de l'enfance, il est mis en liberté surveillée. En mars 1938, à l'âge de 15 ans, il est embauché comme commissionnaire chez Atar, une grande imprimerie genevoise, et abandonne malgré les injonctions judiciaires et médicales la physiothérapie. En juin de la même année, il est accusé de complicité de vol d'accessoires de vélos et, le 16 du même mois, il est placé dans le foyer, dit de semi-liberté [ce qui signifie qu'ils ont la possibilité d'en sortir chaque jour pour faire leur apprentissage ou suivre les cours dans le cadre de leur scolarité], pour "adolescents difficiles ", "Les Ormeaux», situé à Choully, près de Satigny, à une dizaine de kilomètres de la ville. C'est la dernière information reportée dans son dossier.

\section{La voix des maîtres ou chronique d'un cancre annoncé}

3 La première étrangeté pour un chercheur français peu familier du fonctionnement des établissements éducatifs en Suisse est de découvrir l'ampleur du dispositif de contrôle exercé par l'Institution scolaire et son personnel à travers notamment ce service médico-pédagogique, appelé aussi de façon plus explicite service d'observation des écoles, dont est issu ce dossier. Les instituteurs et institutrices non seulement ont un rôle d'évaluateurs des performances et du comportement des élèves au sein de l'école, mais prolongent cette surveillance bien au-delà du temps scolaire.

4 Le premier rapport dont le dossier garde la trace date du 7 novembre 1930. Lucien est sur le point d'avoir 7 ans et vient d'être transféré dans une classe « faible » à plus petit effectif. Le pronostic dressé par sa maîtresse est pour le moins sombre :

"Élève pénible et agité qui bat continuellement ses camarades. Pendant les récréations, il déchire leurs habits et enlève leurs bonnets. Ni gronderie ni punition ne changent en rien sa conduite. En classe travaille de façon très irrégulière, n'a pas d'attention, écriture déplorable, aucun soin dans ses travaux. A raturé son carnet pour effacer le mauvais chiffre de conduite. Fait de façon maladroite qui prouve son 
inconscience. Ce qui frappe chez lui c'est qu'il est distrait, non pas, je crois par méchanceté, mais par anomalie (a un frère en classe spéciale). »

Dans le formulaire de l'enquête scolaire qui se présente sous forme de questionnaire qu'elle remplit parallèlement, elle signale certes qu'il vient régulièrement à l'école et à l'heure, qu'il est propre et bien tenu, mais en revanche tous les autres curseurs sont au rouge : il est " agité », " renfermé ", « inconscient », " insensible aux éloges comme aux blâmes ", "têtu ", "brutal », " distrait ", "babillard ", "sans aucune persévérance ", " n'a de goût prononcé pour rien », "ment instinctivement avant même de savoir de quoi on l'accuse", "rien n'éveille ni ne retient son attention ni son esprit d'observation ", sa mémoire est nulle, son écriture est désordonnée, ses progrès sont très irréguliers et presque nuls, «n'a aucun goût ni application même pour les travaux manuels », a une intelligence anormale et de conclure, comme idée générale qu'elle se fait de l'enfant: «anormal inconscient qui peut devenir dangereux pour ses camarades » en s'inquiétant que cela tienne peut-être de « l'hérédité » car, rappelle-telle encore une fois, il a " un frère en classe spéciale »!

En annexe du rapport est consignée [preuve à l'appui ?] une page du cahier scolaire de l'enfant. Paradoxalement, cette page de cahier semble peu représentative des remarques émises sur l'aspect brouillon et frondeur de l'élève. Elle n'est ni particulièrement raturée ni désordonnée, l'écriture est ronde et appliquée, malgré quelques fautes d'orthographe dûment signalées d'un double soulignement, et le sujet de la rédaction [ou dictée ?] qui figure en haut de la page, au sujet sûrement imposé, offre une vision paisible :

« Nous sommes dans le port de Genève. Des barques quittent la rade, le vent pousse les voiles blanches. Le jeudi, nous allons à la campagne, les alouet montent dans le ciel, l'eau clair chante dans les ruisseaux. Bluets et pavos fleurissent au soleil dans les blé. Grillons noirs et sauterelles vertes jouent dans l'herbe. Nous chassons les papillons aux ailes soyeuses et nous suivons les haies pour trouvez des nids. »

Le second document date du 24 février 1934, peu avant son transfert dans l'école-jardin de Varembé, il s'agit cette fois-ci d'un court rapport manuscrit adressé par son maître, un certain M. Frick, au service d'observation des écoles : l'élève Lucien y est signalé non seulement comme "très désobéissant", «insensible aux conseils comme aux reproches ", avec " un travail en classe insuffisant ", mais aussi et surtout comme un adepte de l'école buissonnière : "Lucien manque aussi la cuisine scolaire et la classe gardienne pour aller s'amuser dans la rue et au bord de l'Arve. » La décision est donc prise de le sortir de «l'école ordinaire».

De son passage à l'école-jardin de Varembé de 1934 à 1936, nous disposons d'un rapport final psychopédagogique. Si son attention est considérée comme «très superficielle et sans durée » et s'il est désigné comme "lent» et ayant un "esprit paresseux» et " dispersé ", en revanche il est considéré comme « observateur ", " très imaginatif » et « débrouillard », doté d'une bonne mémoire "visuelle surtout », il raisonnerait « assez bien » et serait « assez adroit ». En résumé il aurait « des possibilités intellectuelles qu'il n'utilise pas par paresse », se laisserait "rebuter par la moindre difficulté ", mais dans l'ensemble les sensations externes seraient «normales ». De façon très surprenante, ce qui inquiète ce n'est plus son degré d'intelligence ni ses performances scolaires, mais plutôt ses fréquentations en dehors de l'école et son goût immodéré pour les salles obscures. Le 11 mars 1935, le directeur de l'école adresse ainsi une lettre au secrétaire du département de Justice et de la Police pour l'informer des incartades de Lucien, 
cette lettre est accompagnée de la confession de l'enfant écrite sous la dictée de son instituteur [sans aucune faute d'orthographe, il faut bien aussi le souligner] :

« Je devais aller vendredi 8 mars à ma leçon de musique de 6 à 7 heures. Ma mère m'avait donné 5 francs pour payer mes leçons. Je ne suis pas allé à la leçon et je ne suis pas rentré à la maison après l'école, à 6 heures et je n'ai pas soupé. J'ai été cherché J. et nous sommes allés au cinéma Novelty, on jouait La Ruée vers l'Ouest. J'ai payé $2,40 \mathrm{~F}$ pour les deux, la dame ne nous a pas demandé notre âge et personne ne nous a rien dit. J'ai acheté des sibérias [une marque de glaces vendue couramment pendant les entractes dans les salles de spectacles de cette époque] et des caramels au Grand Passage. Nous sommes rentrés à 11 h 15. "

Le 17 janvier 1936, c'est cette fois-ci son instituteur qui adresse un rapport à la Chambre pénale de l'enfance pour signaler les dérives à répétition du comportement de Lucien :

«Grand amateur de cinéma, s'ingénie à pénétrer dans les salles donnant un spectacle interdit aux enfants. S'intéresse uniquement aux films policiers, aux scènes violentes, etc. Assiste aux matchs de boxe, bien que sachant que l'entrée lui en est interdite. À facilement de l'argent en poche sans pouvoir en indiquer la provenance. Beaucoup trop livré à lui-même ; se couche souvent très tard et arrive en classe en retard et trop peu reposé. »

10 Comble de l'étrangeté, c'est finalement la délinquance qui sort définitivement Lucien de la suspicion d'arriération mentale ou même tout simplement scolaire. À partir du moment où il est interpellé pour complicité dans un double vol et interné pendant presque un an à la Maison des Charmilles avec scolarisation intramuros, le regard sur ces performances scolaires change du tout au tout. Le rapport psychopédagogique faisant le bilan de son séjour et daté du 5 janvier 1937 évoque encore son « caractère assez difficile, n'acceptant pas toute observation sans montrer de la résistance, de l'emportement" voire même parfois des "colères promptes et violentes", mais considère qu'il s'agit d'un "élève intelligent, s'intéressant à l'étude et montrant certaines dispositions pour le dessin et les travaux manuels. Mémoire bonne, attention soutenue, s'exprime correctement». Le détail des rapports d'observation mensuels remplis par les instituteurs de mars à décembre confirment cette vision globalement positive : «Bien disposé », « Pourrait avoir avec le temps une bonne influence autour de lui ", "conduite remarquable cette semaine", "sensible", "ronchonne un peu moins ", "très désireux de s'améliorer ", "gros progrès, cherche à se rendre utile ", "Ne hausse pas les épaules à la suite d'une observation ", "très gentil et cherche à rendre service ", " a beaucoup d'empire sur lui-même depuis quelques temps ", "se conduit toujours bien maintenant». Le 7 décembre 1936, Blanche Richard, sa juge assesseur, après lui avoir rendu visite, signale même qu'il « est au tableau d'honneur depuis 3 semaines ».

11 À sa sortie, après avoir réintégré « l'école ordinaire », ce n'est donc plus les capacités de l'élève qui sont en question mais toujours son comportement en dehors de l'école. Dans une lettre du directeur de l'école adressée au service médico-pédagogique, le 17 mars 1937, il est ainsi fait mention de sa conduite qui, bien qu'elle donne totale satisfaction en classe à son instituteur, doit manquer de surveillance le soir puisqu'il a été vu non seulement dans un café, jouant aux cartes avec ses parents, mais aussi dans des combats de boxe, faisant même office de soigneur d'un des boxeurs!

12 L'année suivante, il est orienté, visiblement contre son gré, en école de préapprentissage du Grütli, une école qui accueille chaque année deux à trois cents 
élèves âgés de 12 à 15 ans, autant en fonction de leur manque d'aptitudes pour l'enseignement scolaire que de leur milieu familial modeste.

Depuis l'année antérieure, Lucien réclamait pourtant en vain d'être « libéré » des écoles pour travailler. Cette expression très étonnante vient fortement interpeler le chercheur en sciences de l'éducation et en particulier l'historien français de l'éducation. Cela nous montre tout d'abord les décalages existant selon les pays sur la question de l'obligation scolaire, qui à cette époque en France, rappelons-le, venait tout juste de se prolonger de 13 à 14 ans, alors que dans le canton de Genève [il est important de le préciser, car dans le système fédéral suisse il y a autant de systèmes éducatifs que de cantons!], elle l'est jusqu'à 15 ans depuis 1886. En utilisant l'expression "libéré des écoles", que reprend aussi nous le verrons son propre père, Lucien nous fait ainsi découvrir une autre dimension beaucoup plus contraignante de cette obligation scolaire, à l'encontre de la vision unanimiste et plus politiquement correcte qui la présente comme une des grandes conquêtes républicaines sans tenir toujours compte des décalages avec le ressenti de certains individus ou franges de la population.

14 Faute d'être entendu dans son désir d'émancipation, Lucien va faire preuve de «mauvais esprit», ce qui le conduit à être définitivement classé comme élève perturbateur, arrogant, mauvais élément et meneur, voire comme une graine de voyou, à l'encontre duquel il ferait bon de sévir. Les rapports du directeur de l'école, qui le fustige avec véhémence et réclame châtiment, sont d'autant plus surprenants qu'ils sont signés: Robert Dottrens. Bien que ce dernier soit moins connu en France que certains noms faisant partie du panthéon de l'histoire de l'éducation nouvelle, comme Adolphe Ferrière ou Édouard Claparède, pour les historiens de cette mouvance il est déjà possible de le repérer dès les années 1930 comme pédagogue expérimentaliste. Ancien instituteur, ce dernier avait été un des premiers de ce corps professionnel à être diplômé en pédagogie de l'Institut Jean-Jacques Rousseau, berceau des sciences de l'éducation. En 1931 il était devenu docteur en sociologie de l'université de Genève, sa thèse portant sur Le problème de l'inspection et l'éducation nouvelle: Essai sur le contrôle pédagogique et social de l'enseignement primaire. Il était de plus un des membres fondateurs du Bureau international d'éducation et auteur d'un ouvrage remarqué, publié en 1936, intitulé L'Enseignement individualisé.

Or contrairement à ce que l'on pourrait s'attendre, les écrits du quotidien de ce pédagogue en exercice sur le terrain s'avèrent très éloignés de la théorie. Dans un premier rapport envoyé au directeur de l'Enseignement primaire daté du 7 février 1938, Robert Dottrens semble avoir perdu sa foi en l'« éducation nouvelle " - et en particulier en l'initiative personnelle de l'individu - pour dénoncer avec exaspération le comportement jugé inacceptable de Lucien qui aurait fait preuve dès son arrivée d'un " entêtement opiniâtre ", refusant par principe de suivre les conseils de son maître et n'acceptant d'obéir " qu'à la dernière extrémité ». Il se dispute avec ses camarades et ne se reconnaît jamais coupable; il «trouble les leçons » et «comme il fait figure de meneur dans la classe, il a sur ses camarades une très fâcheuse influence "; ont été saisies sur lui « des publications obscènes »; enfin, prétextant de ses douleurs dans les pieds, il caracole en bicyclette dans l'école et en profite aussi pour faire quelques échappées pour tarabuster les filles de l'école du boulevard James Fazy! Et Robert Dottrens de faire part de sa " conviction très nette qu'il ne tardera pas à mal tourner ", demandant son retrait au plus tôt de l'école. Quelques jours plus tard, alors qu'il a 
prononcé une exclusion temporaire de Lucien de son établissement et que ce dernier continue, semble-t-il, à venir le narguer en faisant des acrobaties sur sa bicyclette devant les grilles de son école, Robert Dottrens envoie un nouveau rapport cette fois-ci au service d'observation des écoles dans lequel il renonce à toute expérimentation pédagogique sur le jeune Lucien, réclamant un «mode d'intervention rapide et énergique » contre cet élément « tout à fait dangereux ». Sa dangerosité est ici évaluée en fonction de la contamination possible des autres élèves par le mauvais exemple :

«Tous nos garçons sont convaincus que Lucien a eu le dessus parce qu'il est renvoyé et ne cherchait que cela et, naturellement bon nombre d'entre eux vont être enclins à utiliser les mêmes moyens pour arriver au même résultat. »

Rien ne semble alors trop dur pour enrailler le mal. Robert Dottrens demande énergiquement des « moyens plus coercitifs » que la procédure de renvoi :

«J'ignore s'il est possible d'organiser en liaison avec l'une ou l'autre des institutions existantes des internements temporaires qui permettraient de sortir les plus mauvais éléments des milieux louches dans lesquels ils puisent leurs inspirations et leurs exemples afin de les rééduquer ou tout du moins de pratiquer à leur égard la cure de désintoxication nécessaire. »

17 Et ce grand pédagogue de proposer une solution pour le moins désuète puisqu'elle fait déjà depuis plusieurs années l'objet, notamment en France, d'une vaste campagne de presse à son encontre: la mise en place "d'un camp agricole de caractère semipénitentiaire » où « nous arriverions à mettre à la raison et à rendre socialement utiles quelques-uns de ces garçons qui, en nous quittant dans quelques mois, vont, sans aucun doute, augmenter la cohorte des individus asociaux desquels nous pouvons tout craindre ». Un «bagne pour enfants» en guise d'expérience pédagogique, drôle de grand écart entre la théorie et la pratique !

\section{Quand la Justice fait langue avec un certain art du contrôle}

18 La seconde surprise nous est venue en feuilletant les nombreux extraits des minutes du Greffe et courriers émanant de la Chambre pénale de l'enfance entre 1936 et 1938. Bien qu'il s'agisse d'un tout autre pays auquel nous sommes peu accoutumés, nous avons ressenti une impression immédiate de familiarité. Comme s'il existait non seulement un même style, les mêmes tournures de phrase, les mêmes énoncés, une même langue internationale des hommes du droit, mais plus encore: un même type de texture de papier et d'en-tête, de tampon, de machine à écrire et de typographie. En litanie sont ainsi égrainées les mêmes formules en ouverture de phrase : les " Vu », « attendu que ", puis les «Ordonne», « dit que »... Les expressions «admonestation», « internement dans une maison d'éducation ou école de réforme", "liberté surveillée ", " amendement ", " huis clos » sont tout aussi familières. Peut-être est-ce dû en partie, nous l'avons appris par la suite, au fait que Genève a été le chef-lieu du « département français » du Léman de 1798 à 1813, date à laquelle il a repris son indépendance pour rejoindre la Suisse.

19 L'imprégnation et la réappropriation basique d'une partie du vocabulaire de la neuropsychiatrie naissante par les hommes du droit, déjà repérées à cette époque en France, semblent aussi concomitantes. Le président de la Chambre pénale de l'enfance 
parlera ainsi «d'insuffisance mentale» et Lucien est désigné à plusieurs reprises comme « instable »; il s'agit d'un « cas mental et éducatif »!

Plus surprenant en revanche nous est apparue l'organisation, dès ces années 1930, d'une justice des mineurs vraiment spécifique qui tardera encore plus d'une quinzaine d'années à se mettre réellement en place en France. Dans le suivi de l'affaire, le dossier témoigne à la fois du fonctionnement d'une Chambre pénale de l'enfance [qui a été instituée dès le 4 octobre 1913 et une révision en 1935 donne plus de place à l'éducation surveillée pour les 10-18 ans], présidée durant toute la période concernée par le même juge, Pierre Jeanneret, mais aussi de façon plus originale d'un réseau auxiliaire de juges assesseurs désignés par le tribunal pour rencontrer régulièrement l'enfant, non seulement pendant toute la durée du placement, mais aussi au-delà, une fois ce dernier rentré chez lui, faisant alors fonction de " délégué à la liberté surveillée ". Dans le cas qui nous concerne, il s'agit d'une femme, $\mathrm{M}^{\text {lle }}$ Blanche Richard ${ }^{2}$, chargée de "s'occuper spécialement du jeune Lucien » ainsi que l'indique un courrier, daté du 9 octobre 1936, de la Chambre pénale au directeur du service d'observation des écoles, Édouard Laravoire. Effectivement, les documents consignés dans le dossier et portant sa signature [B.R. ou BlRichard] témoignent de la très grande persévérance de cette " juge assesseur ». Non seulement, elle soumet Lucien durant son séjour aux Charmilles, à des " interrogatoires" [qui ont en fait plus l'allure d'entretiens compréhensifs] les 28 février, 26 juin, 12 août, 15 septembre, 9 octobre, 7 et 21 décembre 1936, mais aussi elle remplit méticuleusement des fiches de "renseignements para et postscolaires", dans lesquelles elle rend compte régulièrement de toutes les entrevues effectuées au domicile familial de février 1937 à avril 1938. Elle est juge assesseur mais se veut aussi pédagogue et à ce titre, suit les enfants et jeunes "sous mandat d'éducation surveillée ».

21 Plus étonnant encore pour nous a été le faisceau de surveillance extrêmement serré auquel est soumis Lucien en étroite collaboration avec les responsables des différentes institutions scolaires et sous la houlette de ce "service d'observation des écoles ». Il faut croire que le "filet à mailles si serrées », afin qu'aucun cas d'enfant indiscipliné ne pût échapper aux pouvoirs constitués, qu'appelait de ses vœux le conseiller en charge du Département de l'Instruction publique Alexandre Gavard [lors des discussions concernant la loi la déchéance de la puissance paternelle et sur l'enfance abandonnée de 1891] à la fin du XIX siècle, comme le cite l'historienne Martine Ruchat dans son ouvrage L'oiseau et le cachot, a fini par se tisser. Il est frappant de constater qu'à partir du moment où Lucien a été signalé pour la première fois en 1930 au service d'observation des écoles, ce dernier exerce à son égard une vigilance de pratiquement tous les instants durant les huit années suivantes. Non seulement il fait l'objet d'un suivi draconien au sein de l'enceinte scolaire, mais de plus il est pisté et dénoncé par ces mêmes instances pour ses moindres faits et gestes à l'extérieur de l'école, même en dehors du temps scolaire. Ce sont bien ses propres instituteurs qui semblent faire office d'« indics ", non seulement auprès des forces de l'ordre, mais aussi qui se saisissent du système judiciaire, répertoriant ses différentes activités durant la journée et surtout en soirée: sorties cinéma, matchs de boxes ou fréquentation des cafés, même s'il s'agit d'une sortie accompagné de ses parents [soulignant ainsi leur propre faute éducative]. Le dossier comporte ainsi un formulaire pré-imprimé des plus incongrus émanant du Département de l'Instruction publique et intitulé «Discipline hors de l'école ». Ce formulaire rappelle que, dans le cas $d^{\prime}$ '«enfants rencontrés dans la rue», non seulement "pendant les heures de classe» mais aussi «le soir, après les heures 
réglementaires ", il existe un article 118 du règlement de l'enseignement primaire [Et non du Code civil] qui précise qu' 'il est interdit aux enfants non libérés des écoles de se trouver seuls dehors, après 21 heures sans motif légitime ». Dans le rapport de gendarmerie dressé le 5 février 1938 à l'encontre de Lucien, pris en flagrant délit en train «d'importuner » les filles de l'école du boulevard James Fazy [fait d'autant plus grave que les gendarmes arrivés sur place constatent avec inquiétude que « les filles ne faisaient rien pour ne pas attirer ces jeunes gens " et avaient même "une attitude provocante »], il est bien fait mention que l'intervention a été décidée suite à une lettre écrite par le directeur de l'Enseignement primaire.

À l'inverse et de façon tout aussi surprenante, ces enseignants et le responsable du service d'observation des écoles sont interpellés par le président du tribunal. Lorsqu'il a une hésitation à prononcer un relâchement, ce dernier leur demande de donner leur avis même sur un comportement qui ne s'est pas déroulé au sein de l'école; et leur opinion compte et est largement citée dans les comptes rendus d'audience.

\section{Mauvais parents ne sauraient se faire entendre}

Sans surprise en revanche pour ce type de dossier, le cadre familial semble être en Suisse, comme c'est le cas à cette époque pour la France, le premier dans la ligne de mire pour tenter d'expliquer le comportement du garçon. Dès les premiers rapports scolaires la défaillance des parents est pointée du doigt. Tandis que sa collègue de l'école primaire avait déjà évoqué en réponse à l'enquête de 1930, le fait qu'elle n'avait vu qu'une seule fois la mère ainsi que les discordances régnant au sein du couple : « le père plus indulgent que la mère ", M. Frick, dans son rapport de février 1934, exprime son opinion de façon plus tranchée : " J'ai vu le père et la mère qui semblent incapables de remettre leur garçon sur le droit chemin. » À partir de 1936, dès que la Justice s'en mêle, le portrait des mauvais géniteurs devient encore plus explicite en mettant en avant leur « carence éducative ». Dans une première note remise le 17 janvier 1936 à la Chambre pénale, le père est présenté comme "hâbleur, paresseux et buveur ", la mère exerce quant à elle une "mauvaise influence ». Les interrogatoires réguliers auxquels est soumis Lucien par la juge assesseur du tribunal confirment ces premières conclusions : le père «fait des scènes à sa mère, boit trop et donne des coups "; et c'est la mère cette fois-ci qui montre «beaucoup de faiblesse et d'indulgence pour les fautes de son fils", elle surjoue d'ailleurs son rôle de femme éplorée: "Elle prétend que depuis son départ, elle a maigri de $10 \mathrm{~kg}$ ». Le "pauvre gosse » aurait alors avoué en pleurant qu'il était beaucoup plus heureux à la Maison des Charmilles que chez lui. La "mauvaise impression", terme que la juge emploie, est persistante même après la sortie de l'enfant de l'établissement. Dès février 1937, elle évoque les conflits continuels au sein du couple du fait que la mère, " au lieu de soutenir son mari, prend le parti du petit ». En mars 1938, après l'exclusion de Lucien de l'école du Grütli, elle affirme que les "parents ne jouent pas franc jeu ", ce qu'avait déjà souligné le directeur de l'école du Grütli dans une série de lettres, des 6 au 10 février, dans lesquelles il émettait des doutes sur la sincérité du père qui « prétend que son garçon souffre d'une paralysie des pieds et qu'il lui est impossible de marcher ». Il le qualifie d'« homme de fort peu de volonté ", voire qui " trouverait même un certain plaisir à considérer l'attitude de son fils » et affirme que ce dernier aurait reconnu son incapacité à se faire obéir. 

des parents face aux décisions et interventions scolaires et judiciaires, c'est pourtant un tout autre visage qui transparait. Ces parents de " fort peu de volonté ", « incapables de se faire obéir ", qui présentent tous les symptômes de la " carence éducative ", avec un cocktail de brutalité, d'ivrognerie et d'hystérie, voire de désintérêt pour leur rejeton, se montrent pourtant étonnamment pugnaces face au système judiciaire qui préconise pour le «bien de l'enfant" de le "soustraire à sa famille». Les comptes rendus et courriers de la Chambre pénale de l'enfance révèlent en effet l'insistance avec laquelle les parents de Lucien «ne cessent de la poursuivre », tentant tout le long de l'année 1936 de faire recours à la décision de placement, en formulant des «requêtes en libération anticipée » ou tout du moins en demandant de modifier la sentence pour que leur fils soit externe au lieu d'interne de la Maison des Charmilles. Les arguments avancés par le père détonnent par rapport au portrait qui est donné de lui : «il désire en effet que son fils suivent des cours de solfège qu'il a commencé déjà et qu'il suive également les cours du soir d'Allemand». La mère fait quant à elle des visites régulières, parfois même clandestines quand elle a dépassé le quota permis.

Obligée de statuer à chaque fois, la Chambre pénale de l'enfance dans un premier temps déboute systématiquement ces requêtes, après avis négatif du service d'observation des écoles, jusqu'à finalement céder en décembre en prononçant la libération anticipée demandée.

De la même façon, l'année suivante, alors qu'une sorte de liberté surveillée est instaurée, le père fait à nouveau preuve d'un esprit de répartie qui contraste avec le manque de volonté qui lui est attribué. En avril 1937, suite à un nouveau signalement effectué par le service pédagogique, le père semble excédé et se rebiffe devant la juge assesseur venue demander des comptes et qui rapporte avec une certaine stupéfaction ses propos :

"Il dit qu'il en a assez de cette surveillance, qu'il a été à l'école jusqu'à 11 ans, puis qu'il a été libéré et il est aussi cultivé que nous ! Il ne comprend pas qu'on ait fait un rapport au tribunal sur la présence de Lucien dans un café où il jouait aux cartes avec ses parents. Le café dit-il est "l'église du pauvre". Il va faire paraître un article dans La Tribune [Le journal La Tribune de Genève] pour s'en plaindre. »

\section{La voix du sang : un destin tout tracé ?}

La dernière information du dossier nous apprend qu'après avoir été finalement « libéré des écoles » et avoir trouvé du travail dans une imprimerie, Lucien est à nouveau arrêté pour vol et placé dans un nouveau foyer. Rien ne nous permet de savoir ce qu'il est devenu par la suite. Le suivi s'arrête donc bien un jour...

En revanche au fil du dossier, par petites touches se dessinent quelques pistes sur ce qu'il aurait aimé devenir et souvent plutôt, de façon édifiante, sur ce qu'on aurait voulu qu'il devienne. En février 1936, la juge assesseur venue lui rendre visite nous apprend que son père voudrait en faire un agent de sûreté mais que Lucien préférerait être plutôt boulanger ou coiffeur. Le 9 octobre de la même année, Blanche Richard nous informe cette fois-ci que le père de Lucien désire qu'il devienne détective [ce qui n'est pas tout à fait la même chose que policier] mais elle estime que «ce serait mieux pour lui d'avoir un travail manuel, de faire un préapprentissage puisqu'il est habile de ses mains, même s'il n'a aucun goût prononcé ». Le 7 décembre, semblant oublier que 
c'était plutôt un désir du père que le sien, elle semble agacée par son entêtement en affirmant qu'« il vaut mieux qu'il songe à un autre métier que celui de détective » et trouve pour lui l'inspiration héréditaire: il sera menuisier comme son père! L'idée semble d'autant plus bonne que "son père n'a pas de travail et pourra lui apprendre ", peu importe donc que cette perte d'emploi soit un signe que le secteur est peut-être en crise. Nous pouvons alors légitimement nous demander de quel apprentissage s'agit-il : menuiserie ou chômage?

\section{BIBLIOGRAPHIE}

DROUX Joëlle, RUCHAT Martine, « L'« enfant-problème », ou l'émergence de figures problématiques dans la construction d'un dispositif de protection de l'enfance (Genève, 1890-1929) », Carnets de bord, $\mathrm{n}^{\circ} 14,2007$, p. 14-27.

KLOos Grégory, L'Hospice général dans les années 1940 et 1950 : le discours sur l'assistance et les personnes assistées, Mémoire de DEA en histoire économique et sociale sous la direction de A.-L. Head, Université de Genève, mars 2003.

MARCACCI Marco, CHARDON Elizabeth, Tu finiras au Grütli ! Une école de fin de scolarité. Genève, 1929-1969, Genève, La Criée/Éditions Passé Présent, 2004, 200 p.

PETITAT André, « Inégalités scolaires et inégalités sociales : le cas de Genève », dans Production de l'École - Production de la société. Analyse socio-historique de quelques moments décisifs de l'évolution scolaire en Occident, Genève, Librairie Droz, coll. « Travaux de Sciences Sociales ", 1999, p. 365-413.

RUCHAT Martine, « Raisons disciplinaires, écritures cliniques et figures de l'enfant dans l'histoire de l'éducation spéciale 1867-1948», Studien und Quellen, n² 29, 2003, p. 243-264.

RUCHAT Martine, L'oiseau et le cachot : Naissance de l'éducation correctionnelle en Suisse romande 1800-1913, Genève, éditions Zoé, 1993.

\section{NOTES}

1. Mais c'est aussi qu'en Suisse c'est la commune d'origine qui paie pour ses ressortissants. Je remercie Martine Ruchat pour cette précision.

2. Blanche Richard, fille et sœur de professeurs de droit, avait fondé dès la Première Guerre mondiale une association Les volontaires du service social pour venir en aide à l'enfance difficile. De 1935 à 1936, elle fut nommée juge assesseur à la Chambre pénale de l'enfance, par cette élection, elle devient la première magistrate judiciaire de notre pays. Voir article "Plus de 50 ans d'aide à la jeunesse difficile et inadaptée " écrit à l'occasion de son $80^{\mathrm{e}}$ anniversaire dans Femmes suisses et le Mouvement féministe, organe officiel des informations de l'Alliance de Sociétés Féminines Suisses, juillet 1964, p. 3. 


\section{RÉSUMÉS}

Le dossier du service médico-pédagogique de la ville de Genève sur le jeune Lucien datant des années 1930 est surprenant à plus d'un titre. Il démontre tout d'abord l'ampleur de la surveillance exercée par l'école et ses maîtres sur la vie des élèves, non seulement pendant le temps scolaire, mais aussi en dehors faisant penser à un contrôle social des milieux populaires. Dans le même temps, il révèle la mise en place très précoce d'un suivi judiciaire personnalisé par l'intermédiaire de juges assesseurs qui plus est femme. S'il s'avère très similaire en revanche aux dossiers français sur la question du regard stigmatisant porté sur les familles, il nous réserve sa part de surprise dans ce qu'il laisse percevoir de la réaction de ces dernières face à ce jugement.

The file of the medico-educational service of the city of Geneva on the young Lucien dating from the 1930s is surprising in more than one way. First of all, it shows how the school and its teachers are monitoring the lives of their student, not only during school time, but also outside, suggesting that social control of the working-class is being exercised. At the same time, it reveals the very early implementation of a personalized judicial follow-up through the intermediary of judge assessors, who is and in this case a woman. On the other hand, although it is very similar to the French files on the issue of the very stigmatizing way of looking at families, but it reserves us its share of surprise in what it suggests the reaction of those families to this judgment.

\section{INDEX}

Index géographique : Suisse, Switzerland

Mots-clés : justice des mineurs, dossiers de jeunes, récit de vie, service médico-pédagogique

Keywords : juvenile justice, case files, life story, child guidance service

\section{AUTEUR}

\section{MATHIAS GARDET}

Historien, professeur en sciences de l'éducation, Université Paris 8, membre du CIRCEFT. 